International Journal of Case Reports
(ISSN:2572-8776)

\title{
Burkitt Lymphoma of Central Nervous System in an elderly-patient: A new approach with a modified classic regimen
}

Maria Cynthia Fuentes-Lacouture ${ }^{1}$, Camila Torres Vélez ${ }^{2}$, Sergio Alejandro Osorio ${ }^{2}$, Mariafernanda Gonzalez-Blanco', Angélica Ovalle ${ }^{2}$, Juan Sebastian Salas Botero ${ }^{3}$, Yadira Vasquez $^{3}$, Claudia Patricia Niño ${ }^{2}$ Javier Segovia ${ }^{2}$

${ }^{1}$ Medicina Interna, Universidad del Rosario. Hospital Militar Central. Bogotá D.C. Colombia. ${ }^{2}$ Departamento de Hematología y Oncología. Hospital Militar Central. Universidad Militar Nueva Granada. Bogotá D.C. Colombia. ${ }^{3}$ Departamento de Patología. Hospital Militar Central. Universidad Militar Nueva Granada. Bogotá D.C. Colombia.

\section{ABSTRACT}

Burkitt's lymphoma $(B L)$ is one of the high-grade lymphomas, *Correspondence to Author: characterized by a rapid growth. They are usually treated with María Cynthia Fuentes Lacouture intensive chemotherapy regimens, being normally chemo-sensi- Department: Internal Medicine, Unitive, but at the expense of high toxicity secondary to treatment. versidad del Rosario. Transversal Additionally, the compromise of the central nervous system (CNS) 3C \#49-02 Bogotá D.C. Colombia. implies a major risk as well as greater toxicity, taking into account Tel: (+57) 3112430465 a worse clinical prognosis with a requirement of more intensive schemes to achieve control of the disease. This implies doubts in the management of older patients with BL with CNS comHow to cite this article:

promise, in whom toxicity is a limitation to these therapies, and there are no other alternatives that offer better benefit in terms of less frequent or severe adverse events, with similar outcomes in terms of progression-free survival (PFS) or overall survival (OS). Therefore, clarifying cases such as the one we report below allows us to provide a therapeutic alternative for older or unfit patients, in whom the intention of treatment should be to seek a good tumor response, but without ignoring the potential toxicity of chemotherapy.

Maria Cynthia Fuentes-Lacouture, Camila Torres Vélez, Sergio Alejandro Osorio, Mariafernanda Gonzalez-Blanco, Angélica Ovalle, Juan Sebastian Salas Botero, Yadira Vasquez, Claudia Patricia Niño, Javier Segovia. Burkitt Lymphoma of Central Nervous System in an elderly-patient:A new approach with a modified classic regimen. International Journal of Case Reports,

Keywords: Burkitt lymphoma, central nervous system lympho- 2021,5:244. ma, elderly, chemotherapy. 


\section{Introduction}

Treatment of high-grade lymphomas is based on intensive chemotherapy regimens. However, the adverse effects and complications of these schemes sometimes limit their initiation, and more so when it comes to unfit patients or older adults. We present the case of an elderly patient, with limited performance status, who debuted with Burkitt's lymphoma in the central nervous system, in whom the choice of therapy represented a challenge due to its inherent risk, and with whom it was achieved an adequate tumor response at the expense of a good safety and toxicity profile with an age-adjusted modified regimen of chemotherapy.

\section{Case description}

A 76-year-old female patient with a history of essential arterial hypertension, user of a pacemaker due to node disease and dyslipidemia, consults the emergency department due to the appearance of a mass in the frontal region of the head, which had been growing for the last 5 months. She referred also mild intermittent headache, palpebral edema and loss of $5 \mathrm{~kg}$, without night sweating or fever. Patient was initially assessed by the neurosurgery department, who biopsied the lesion and discharged. After 15 days, she was re-admitted to the emergency room because of progressive and accelerated growth of the mass. On physical examination, the mass was hard, not mobile, and there was no redness or heat. At that time patient had neurological deterioration, with the appearance of enuresis, disorientation and drowsiness. She was hospitalized and a head CT was requested, which showed a mass of an exophytic and apparently endophytic soft tissue component, of subdural morphology, with high suspicion of neoplastic versus infectious origin, associated with vasogenic edema of the brain parenchyma (Figure 1).

Treatment with steroids was initiated with which there was improvement in neurological symptoms. The biopsy report was obtained, which showed infiltration in a diffuse pattern by atypical lymphoid cells of large and intermediate size, with irregular shapes. Immunophenotype showed B cells positive for CD20, with intense co-expression for $\mathrm{CD} 10$, heterogeneous for BCL6 and negative for BCL2, with expression of the protein C-MYC in $20 \%$. Cells where negative for MUM1 and CD30. There was a companion $T$ population positive for CD3, CD5 with a predominance of CD4 over CD8, negative for ALK1, with CD23 positive ruptured follicular dendritic cell networks (figure 2). The Ki67 cell proliferation index was $90 \%$. The study for the identification of the Epstein Barr virus was negative. Systemic HIV test was also negative. A definitive diagnosis of stage IV - HIV negative - Burkitt's lymphoma was made. Fluorescence in situ hybridization (FISH) for $\mathrm{t}(8 ; 14)$ was negative. Neck, chest, and abdomen CT were negative for additional neoplastic disease, and the bone marrow biopsy showed no lymphoma infiltration. Complete blood count showed 5650 leukocytes $\quad(65 \%$ neutrophils, $18 \%$ lymphocytes), hemoglobin $12 \mathrm{gr} / \mathrm{dL}$, hematocrit $36 \%$ and 284.000 platelets. Lactic dehydrogenase was 228. Renal and liver function tests were normal, and there was no tumor lysis syndrome.

Given her age and comorbidities, oncology board decided to initiate management with HyperCVAD, with adjusted doses of cytarabine ( $1 \mathrm{gr} / \mathrm{m} 2 /$ day on days 1 and 2) to reduce the risk of complications and starting with block $B$ to grant the benefit of management with methotrexate given the compromise of central nervous system, associated with intrathecal therapy with methotrexate. With adequate tolerance, patient completed the first cycle, achieving a $90 \%$ resolution of the mass after block B (figure 3). Therefore, the patient was defined as a candidate to continue the proposed chemotherapy regimen.

\section{Discussion}

Burkitt's tumor (or Burkitt's lymphoma) - BL - is part of high-grade non-Hodgkin's lymphomas. Three subtypes are known: sporadic (more frequent in children and predominantly in the 
abdomen), endemic (typical of equatorial Africa and associated almost in all cases with Epstein Barr virus infection and with typical mandibular involvement) and the third, associated with immunodeficiency, typically HIV, but with any state of immunocompromise related to its appearance ${ }^{[1]}$.

Despite the differences in the demographic classification, histologically it is characterized by presenting cells in the appearance of a "starry sky", accompanied by the presence of benign histiocytes. The cells of BL are usually of clear and dispersed cytoplasm, basophilic, and are located in a background of mixed cells (tumor and stromal). The proliferation rate is characteristically high in $\mathrm{BL}$, which confers greater aggressiveness ${ }^{[2]}$.

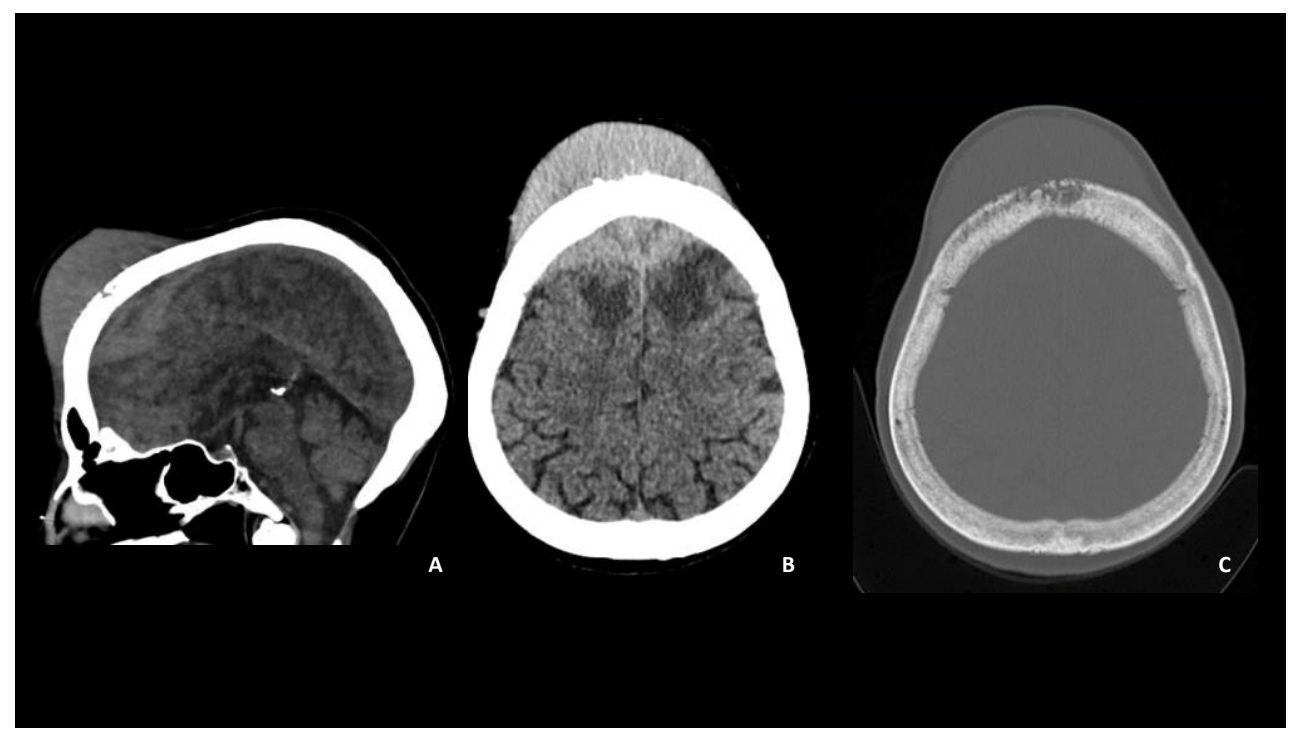

Figure 1. Pre-treatment lesion. A. Sagittal view. B. Coronal view. C. Coronal view, bone enhancement. Dense heterogeneous-looking mass of the skull, mainly in the frontal bone, with a permeative aspect of the external bone towards the midline, with apparently soft tissue component. Significant bilateral superior frontal vasogenic edema.

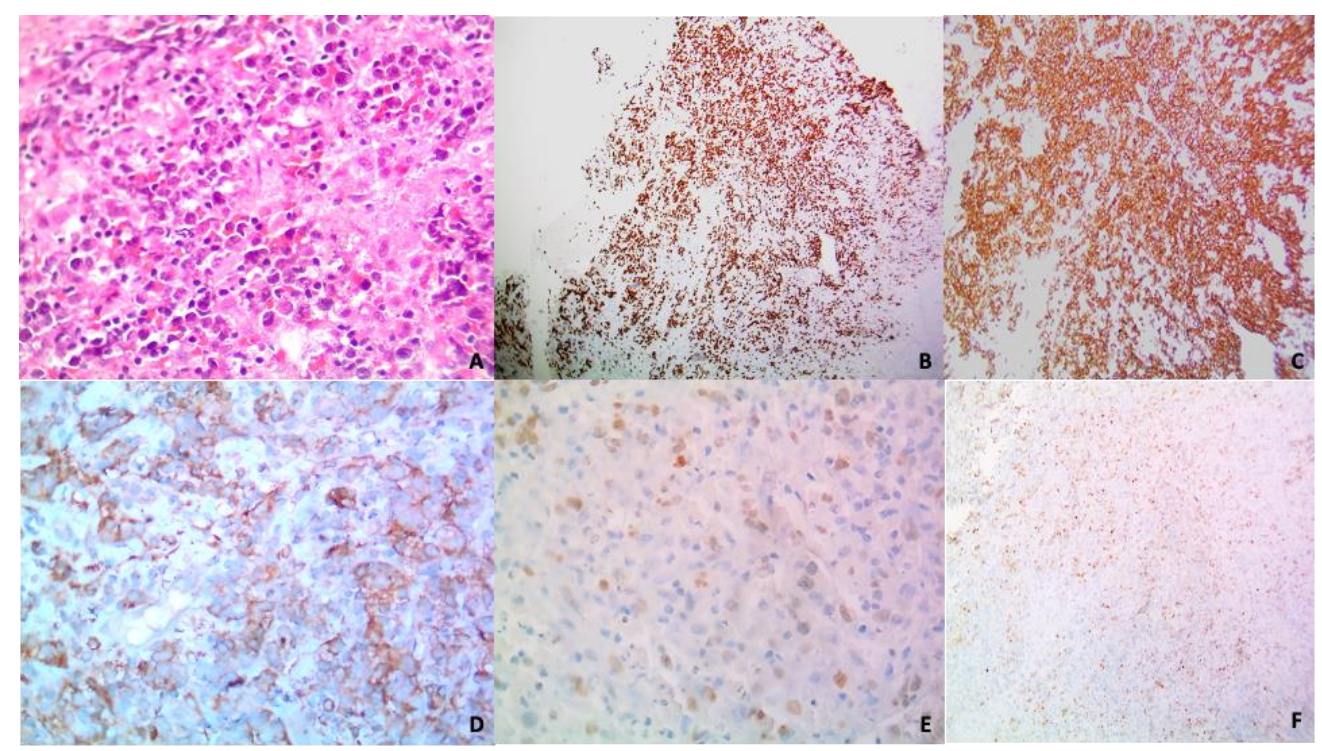

Figure 2. A. H\&E 10X. Tissue fibrosis with presence of a diffuse infiltrate with atypical large and intermediate-sized lymphoid cells of irregular shapes B. 10X. Ki67 90\% in tumor cells C. Tumor cells with Strongly positive CD20 D. Heterogeneous expression of CD10 in tumor cells. E. Heterogeneous expression of BCL6 in tumor cells F. cMYC with diffuse positivity in $20 \%$. 


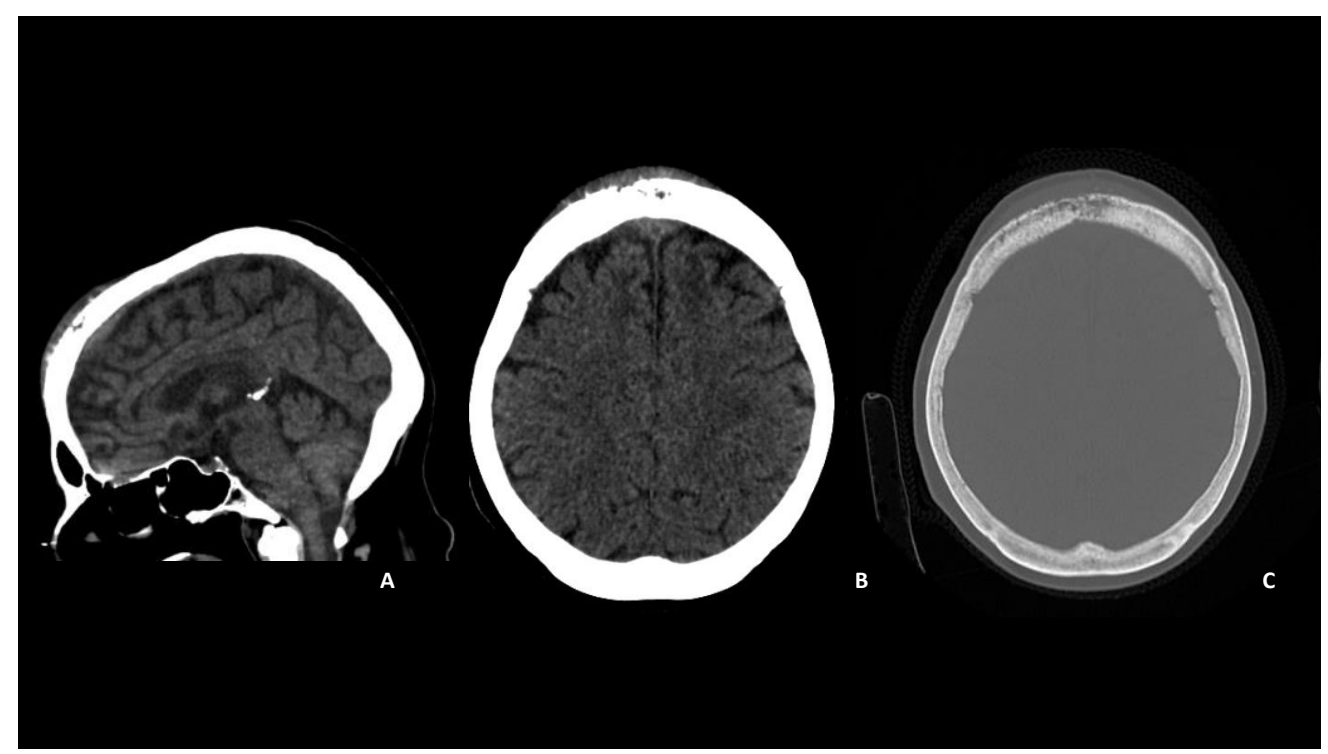

Figure 3. After first cycle lesion. A. Sagittal view. B. Coronal view. C. Coronal view, bone enhancement. Reduction of approximately $90 \%$ of the exophytic lesion evidenced in the previous image, with an important decrease in vasogenic edema. Persistence of changes due to frontal bone osteolysis.

As it is a high-grade lymphoma, the therapeutic recommendation has been based on intensive regimens. Among the available options, those that have shown better results are Hyper CVAD, CODOX-M / IVAC or DA-EPOCH, all with the addition of Rituximab, and all accompanied by intrathecal chemotherapy [3]. The aforementioned is true in young patients, or fit adults, with the ability to tolerate the high rate of toxicity inherent in the aforementioned schemes. In older adults the story is different, taking into account the frailty of these patients, which limits the therapeutic offer due to the higher risk of toxicity and adverse events related to the treatment.

In addition to age, the other factor of poor prognosis in $\mathrm{BL}$ is the compromise of the central nervous system (CNS) taking into account it worsens disease-free survival, as well as it confers a lower overall survival when compared to adults with BL without SNC involvement. This was demonstrated by an important multicenter study, where it was observed that the progression-free survival (PFS) was 69\% vs. $46 \%$ in patients with BL with and without CNS involvement, respectively. Similarly, overall survival (OS) fell from $74 \%$ to $49 \%$, respectively [4]. This difference not only reflects a greater aggressiveness of the disease, but also the limitation in the possible chemotherapy regimens that can be offered to these patients, since not all include drugs with penetrance to the CNS.

In our case, our patient not only debuted with a mass that compromised CNS, but also at 76 years of age with a decreased performance status (secondary to the development of lymphoma which limited patient daily activities and autonomy). All this conferred a challenge on the therapy to choose, taking into account the high risk of complications inherent to the treatment, associated with a high probability of therapeutic failure due to the limitation in the treatments that we could offer.

Among the best options, DA-EPOCH-R has limited penetrance to the CNS given the absence of drugs such as methotrexate and cytarabine. Among the remaining options, Zayac et al showed that the cumulative incidence of CNS recurrence is similar between Hyper CVAD and CODOX-M / IVAC [4]. However, in our institutional experience, the toxicity profile of 
HyperCVAD is lower, and as our patient was an elderly adult, this was the major determinant in defining the therapy to be given. Finally, to reduce myelosuppression and other adverse effects, the dose of cytarabine was reduced according to age, as has been proposed in some recent reviews ${ }^{[5]}$.

At present, our patient continues the following cycles of chemotherapy with HyperCVAD with adjusted dose of cytarabine, with a single complication of mild non-suppurative soft tissue infection. The lymphoma continues in remission.

The excellent clinical results obtained in this case, make us as physicians to present Hyper CVAD with age-adjusted dose of cytarabine as a favorable therapeutic alternative with promising outcomes for unfit or elderly patients. We believe this case represents a support in decision-making in future difficult cases like this, where it should seek a balance between the risk and the benefit of the treatment to be offered.

\section{Conclusion}

Burkitt's lymphoma corresponds to the group of high-grade lymphomas, it requires management with intensive chemotherapy regimens, which usually carry a high rate of toxicity. CNS involvement is common and confers a poor prognosis. The appearance of these two circumstances (high-grade lymphoma with CNS involvement) in elderly patients with limited performance status becomes a therapeutic challenge for the treating physician, since a balance must be found between the benefit of the therapy and the toxicity given to the patient. In our case, we present a feasible and promising therapeutic alternative, with dose modifications that improve the safety profile of the scheme, while continuing to offer good clinical outcomes for older patients who debut with this disease.

\section{Acknowledgments}

None to declare.

\section{Financial Disclosure}

No funding was received. None of the authors have disclosures relevant to this manuscript.
None to declare.

\section{Informed Consent}

The manuscript has been sufficiently deidentified to protect the patient. Patient signed written informed consent for the publication of the case.

\section{Author Contributions}

MCFL and CT wrote the manuscript. All authors contributed to the editing of the manuscript.

\section{References}

[1]. Kalisz, K., Alessandrino, F., Beck, R., Smith, D., Kikano, E., Ramaiya, N. H., \& Tirumani, S. H. (2019). An update on Burkitt lymphoma: a review of pathogenesis and multimodality imaging assessment of disease presentation, treatment response, and recurrence. Insights into imaging, 10(1), 56.

[2]. Dennis P. O'Malley, Aaron Auerbach, Lawrence M. Weiss; Practical Applications in Immunohistochemistry: Evaluation of Diffuse Large B-Cell Lymphoma and Related Large BCell Lymphomas. Arch Pathol Lab Med 1 September 2015; 139 (9): 1094-1107

[3]. Dunleavy K. Approach to the Diagnosis and Treatment of Adult Burkitt's Lymphoma. J Oncol Pract. 2018 Nov;14(11):665-671. doi: 10.1200/JOP.18.00148. PMID: 30423267.

[4]. Adam Zayac, Andrew Matthew Evens, Andrzej Stadnik, Stephen D. Smith, Deepa Jagadeesh, Lori A. Leslie, et al. Outcomes of Patients with Newly-Diagnosed Burkitt Lymphoma (BL) and Central Nervous System (CNS) Involvement Treated in the Modern Era: A Multi-Institutional Real-World Analysis. Blood 2019; 134.

[5]. Rausch, C.R., Jabbour, E.J., Kantarjian, H.M. and Kadia, T.M. (2020), Optimizing the use of the hyperCVAD regimen: Clinical vignettes and practical management. Cancer, 126: 1152-1160.

\section{Conflict of Interest}

